\title{
Acoustic Method for Measuring the Density of Particles in Water
}

\author{
Qiang $\mathrm{Tu}^{\dagger}$, Fei Yuan ${ }^{\dagger}$, Congren Lin*†, En Cheng ${ }^{\dagger}$, Jianghui $\mathrm{Li}^{\ddagger}$ \\ ${ }^{\dagger}$ Key Laboratory of Underwater Acoustic Communication and Marine Information Technology (Xiamen University) \\ Ministry of Education, Xiamen, China \\ ${ }^{\ddagger}$ Institute of Sound and Vibration Research (University of Southampton) \\ Southampton, United Kingdom \\ ${ }^{*}$ Corresponding Author : lincongren@126.com
}

\begin{abstract}
The acoustic technology has a great potential to measure the concentration of particles in water which is one of the parameters for understanding ocean ecology. To achieve this purpose, the forward acoustic propagation model has been considered as a method to obtain concentration and particle size. The Gaussian lognormal distribution was used to describe the particle matters condition in suspended water. Base on the model, a nonlinear inversion process took values of attenuation into calculation and get parameters of concentration, average radius and variance.
\end{abstract}

Index Terms-suspended sediment concentration, acoustic propagation, attenuation

\section{INTRODUCTION}

The suspended particles concentration of ocean or river in hydrology is related to the amount of sediment transported, and also is correlative with the underwater organisms living environment in natural ecosystems. Traditional methods to sample physical liquid, including artificial operating and semi-automatic equipment, are usually laborious and timeconsuming in implementation with a long time interval and small number. However the natured water flow and the artificial events make the parameter of suspended particles varying quickly and frequently. Compared with that, acoustic measurement systems is simpler, faster and more effective with acoustic equipments under water [1] [2].

In suspension, the existence of particulate matter, including inorganic and organic matter, attenuates sound energy received by sensor. This means that particles concentration is related to acoustic attenuation, which can be measured easily. Although there are various reasons causing attenuation, the existence of particulate can be a significant one. Literatures [6] [7] suggest that concentration and particle size can be analysis by studying attenuation.

Initial studies [3] considered the viscous absorption when wave length is larger than particle matter size based on Sewells work [8]. Allegra and Hawley [4] also discussed absorption phenomenon when ultrasonic passing suspensions of small spherical particles. Base on Uricks work [3], Richards [5] proposed a model about acoustic attenuation in suspensions

This work was supported by the National Natural Science Foundation of China $(61471308,61571377,61771412)$ when multiplication of wave number and particle diameter less than one. However, most of these work are only considered average particle radius which is difficult to simulate real particle matters accurately. Due to suspended particle matters are not a uniform material of size and physical composition [8] [9].The existence of small or big particles distinctly obstructs the accuracy of theories with average size. To solve this, one method is to use particle size distribution. Various adoptable distribution models [8] [10] have been proposed, and Stavn and Keen had good summary and deep analysis [11].

The purpose of this paper is to measure the concentration of suspended particles using forward acoustic propagation theory in sea. The discussion of particle size distribution considers new parameters replacing radius and makes the propagation model more effective.

\section{Acoustic AtTEnuation MOdel}

This section constructs an acoustic forward propagation model in suspended liquid. When the sound spreads in the suspended liquid, the sound intensity is decreasing with the increasing distance. This is expressed as attenuation coefficient $\alpha$ which means the ratio of the received sound intensity to the transmitters.

When considering acoustic forward propagation model, attenuation factors include viscous absorption, scattering attenuation, water absorption, geometrical attenuation and heat conduction. However, heat conduction absorption is usually neglected, due to it's insignificant effects. Different from the light wave, acoustic wave frequency is much lower. Therefore, the wavelength is much larger than size of suspended particles which means the scattering happened in sphere particles are mainly Rayleigh scattering [13]. In this case, the scattering attenuation [3] can be computed as:

$$
2 \alpha_{s}=N \frac{4}{9} \pi k^{4} R^{6},
$$

where $k$ is the wave number, $\mathrm{R}$ is the radius of particles, and $N$ is the number of particle matters related to concentration.

However, the existence of relative motion between particle matters and water, which is related to acoustic vibration, also produces viscous absorption and transforms sound power to 


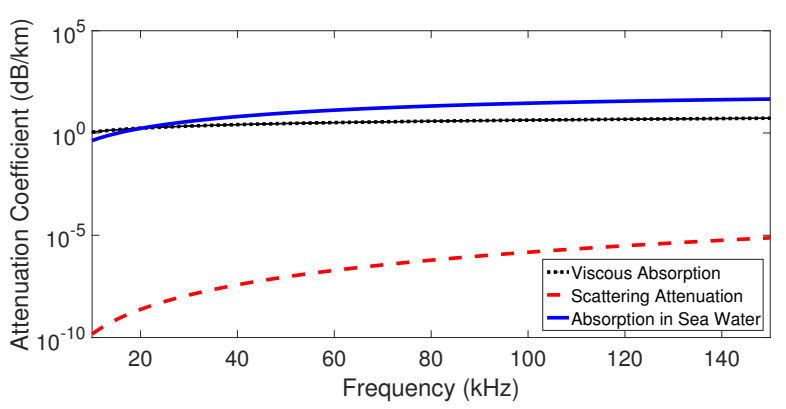

Fig. 1. Attenuation or absorption related to frequency.

heat. Compared to the scattering attention, viscous absorption is several orders of magnitude larger than it. Therefore, the viscous absorption is one of the main factors when considering forward propagation as well as water absorption. In this work, the expression [3] [5] can be:

$$
\begin{gathered}
2 \alpha_{v}=C k(\sigma-1)^{2} \frac{s}{s^{2}+(\sigma+\tau)^{2}} \\
s=\frac{9}{4 \beta \alpha}\left(1+\frac{1}{\beta R}\right), \tau=\frac{1}{2}+\frac{9}{4 \beta \alpha}, \sigma=\frac{\rho_{1}}{\rho_{0}}, C=\frac{4}{3} \pi R^{3} N
\end{gathered}
$$

where $\rho_{1}$ and $\rho_{0}$ are the densities of particles and water, $\beta=$ $\sqrt{\omega / 2 \nu}, \nu$ is viscosity coefficient of background liquid, $\omega$ is the angle frequency of acoustic signal.

As for water absorption, the classical theory proposed by Schulkin and Marsh [12] is used:

$$
2 \alpha_{w}=\left(A \frac{S f_{T} f^{2}}{f_{T}^{2}+f^{2}}+B \frac{f^{2}}{f_{T}}\right) \times(1-C P) .
$$

The geometric attenuation is considered as spherical wave propagation attenuation that expressed as

$$
2 \alpha_{g}=20 \lg r \text {. }
$$

So, the model is expressed as [5]

$$
\alpha=\alpha_{w}+\alpha_{v}+\alpha_{s}+\alpha_{g},
$$

where $\alpha_{w}$ is water absorption coefficient, $\alpha_{v}$ is viscous absorption coefficient and $\alpha_{s}$ is scattering attenuation coefficient measured from acoustic generator to the receiver and $\alpha_{g}$ is geometrical attenuation. Fig 1 and Fig 2 show the variation of four kinds attenuation's variation at different frequencies or distance.

\section{Distribution OF PARTICLE SIZE}

In the previous section, the equations about scattering attenuation and viscous absorption are functions of average particle radius. The particle matters in suspension contain broad size and dominated by sediments near the seabed [14]. It is noteworthy that attenuation of suspension is significantly affected by variations in particle size [15] [16] [17]. For larger particles, it is more effective to sound than that of small particles when calculating $k R$ in equations. Fig 3 shows the viscous absorption coefficient raise and fall for particle radius

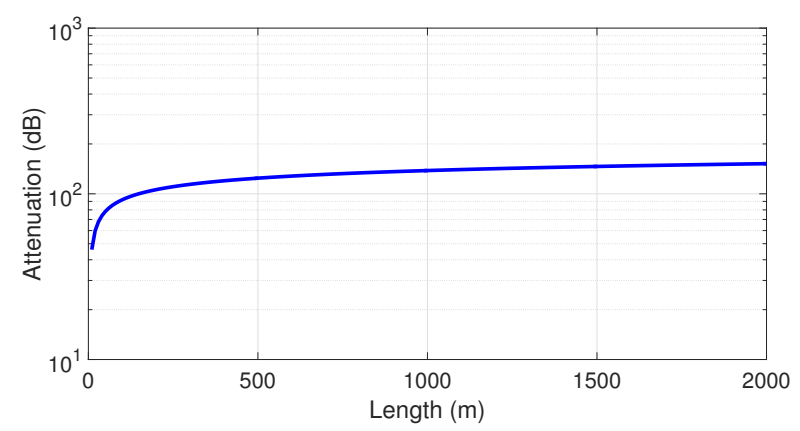

Fig. 2. Geometrical attenuation related to distance.

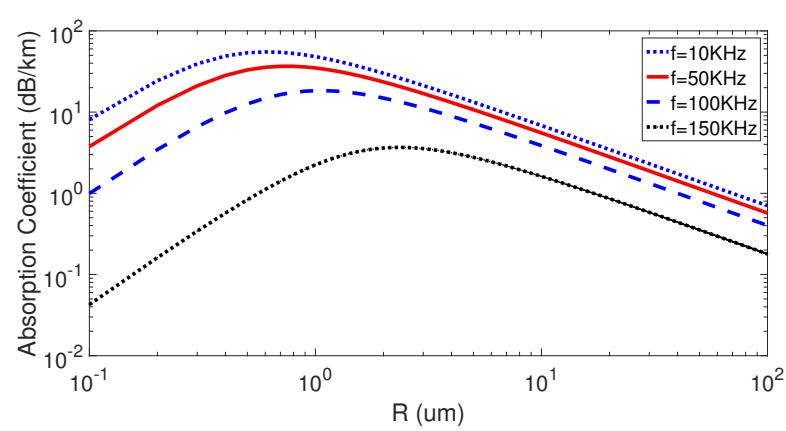

Fig. 3. Scattering attenuation and viscous absorption varying with particle size.

increasing gradually which exist peaks for different frequency. Hence, it is necessary to consider an assumption of particle size to make the theory closer to real situation.

With high-precision optical particle size analyzer [18] [19] or acoustic Doppler current profilers [20] [21], the simples from the several major sea and river have been analyzed. Many possible mathematical modes of particles distributions are summarized [22], and a popular one is the model of particle size corresponding to particle number. Then the particle distribution is expressed by function of particle radius and the Gaussian lognormal distribution is usually used [11]. In this work, [24] is considered as particle size distribution and this description is showed in Fig 2. Assume particles radius

$$
R=2^{-\phi-1} \times 10^{-3} m,
$$

where $\phi$ obeys Gaussian distribution and the probability of $\phi$ is

$$
P(\phi)=\frac{1}{\sqrt{2 \pi} Q} e^{-\frac{(\phi-\bar{\phi})^{2}}{2 Q^{2}}},
$$

where $\bar{\phi}$ is calculated by average particle radius, and $Q$ is the variance. Thus, the number $n_{i}$ of particle matters for a special radius $R_{i}$ is

$$
n_{i}=N P\left(\phi_{i}\right),
$$

and Fig 4 shows the curve of $n_{i}$ and $R_{i}$. 


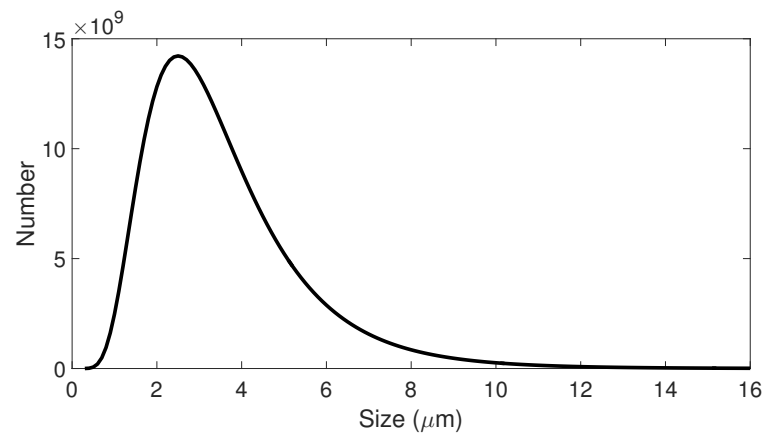

Fig. 4. The particle size and its number of existence in suspension.

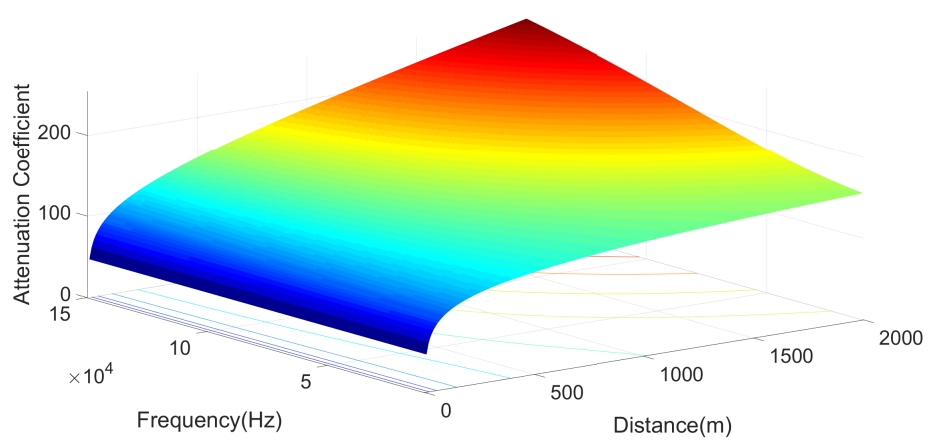

Fig. 5. The acoustic attenuation value related to frequency and spreading range, when considering particle size distribution.

Then, when considering the distribution, the formulas of viscous absorption and scattering attenuation change are given by

$$
\begin{array}{r}
2 \alpha_{s}=\int_{-\infty}^{\infty} N P\left(\phi_{i}\right) \frac{4}{9} \pi k^{4} R\left(\phi_{i}\right)^{6} d \phi_{i}, \\
2 \alpha_{v}=\int_{-\infty}^{\infty} \frac{4}{3} \pi R\left(\phi_{i}\right)^{3} N P(\phi i) \\
\times\left[k(\sigma-1)^{2} \frac{s\left(\phi_{i}\right)^{2}}{s\left(\phi_{i}\right)}+\left(\sigma+\tau\left(\phi_{i}\right)\right)^{2}\right] d \phi_{i} .
\end{array}
$$

It is seen that the probabilities of different radius are calculated and represent the existent number of particles in equations. According to the equations, the acoustic attenuation value related to frequency and distance when consider particle size distribution is showed in Fig 5.

\section{PARAMETER INVERSION}

In the equation, two parts can be separated from attenuation coefficient, one is $\alpha_{s}+\alpha_{v}$ affected by particle matters, the other is $\alpha_{w}+\alpha_{g}$ being independence. For the purpose of measuring particle concentration, $\alpha_{s}+\alpha_{v}$ needs to be leave and others compensated.

$$
\alpha_{f}=\alpha-\alpha_{w}=\alpha_{s}+\alpha_{v}=\int_{-\infty}^{\infty} F_{f}(R(\phi)) P(\phi) d \phi,
$$

where $F(R)_{f}$ is coefficient of scattering attenuation and viscous absorption when $d \phi$ is $\phi$ and $f$ is frequency. So
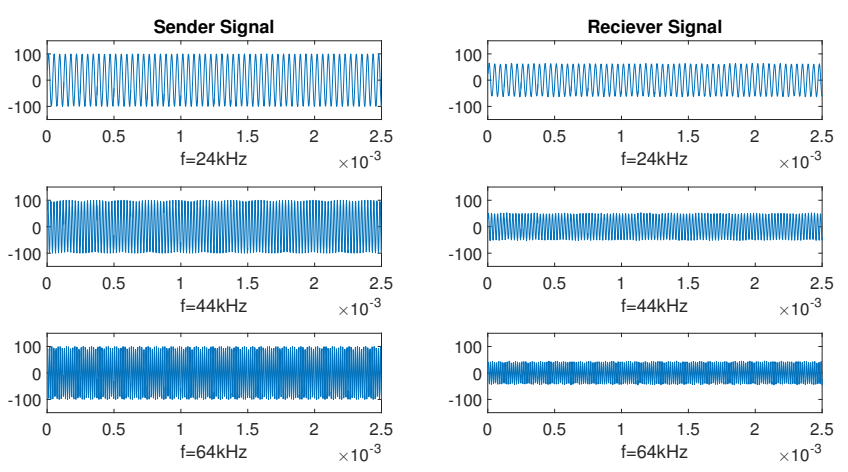

Fig. 6. Signal time-domain diagrams of sound source and receiver when frequencies are $24 \mathrm{kHz}, 44 \mathrm{kHz}$ and $64 \mathrm{kHz}$.

TABLE I

INVERTING PROCESS

\begin{tabular}{|c|c|c|c|c|c|}
\hline Noise & Step & $\mathbf{C}$ & $\mathbf{R}$ & $\mathbf{Q}$ & $\boldsymbol{\phi}$ \\
\hline & 0 & 30 & 0.0160 & 2 & -5 \\
& 100 & 0.6662 & 0.0358 & 2.1382 & -6.1630 \\
$1 \mathrm{~dB}$ & 1000 & 0.5080 & 2.3830 & 0.7367 & -12.2185 \\
& 5000 & 0.5080 & 2.3830 & 0.7367 & -12.2185 \\
\hline \multirow{5}{*}{$5 \mathrm{~dB}$} & 0 & 30 & 0.0160 & 2 & -5 \\
& 100 & 0.0420 & 0.0420 & 2.0966 & -6.3912 \\
& 1000 & 0.5201 & 2.0964 & 0.8035 & -12.0337 \\
& 5000 & 0.5201 & 2.0964 & 0.8035 & -12.0337 \\
\hline $10 \mathrm{~dB}$ & 5000 & 0.5299 & 1.8480 & 0.8645 & -11.8517 \\
\hline \multicolumn{2}{|c|}{ Real } & $\mathbf{0 . 5}$ & $\mathbf{2 . 5}$ & $\mathbf{0 . 7 0 7}$ & \\
\hline
\end{tabular}

the equation about sound frequency and acoustic propagating attenuation in suspension is the prior equation for inverting parameter of particles concentration. The vector $m^{T}=[C, \bar{\phi}, Q]$, where $C$ is concentration of suspension, $\bar{\phi}$ and $Q$ are average and variance of $\phi$. Define nonlinear option operator

$$
G(m)=A=\left[\alpha_{f_{1}}, \ldots, \alpha_{f_{j}}\right]
$$

where the quantity of $j$ is determined by the number of different frequency signals and is far more than the number of variables. Then the main function is built as

$$
\min L(m)=\|G(m)-A\|^{2} .
$$

For approximating optimal solution, the iterative equation is defined as

$$
m_{k+1}=m_{k}-\tau_{k} G^{\prime}\left(m_{k}\right)^{*}\left[G^{\prime}\left(m_{k}\right) G^{\prime}\left(m_{k}\right)^{*}\right]^{-1} \cdot\left(G\left(m_{k}\right)-A\right)
$$

\section{Simulation Results}

This section simulates a bistatic transceiver experiment of the forward propagation model with broad frequencies and gets estimated concentration by iterations of inversion. The acoustic signals are sinusoid signals whose amplitude is 100 and frequencies are from $1 \mathrm{KHz}$ to $150 \mathrm{KHz}$.For testing robustness, two white Gaussian noise of $1 d B$ and $5 d B$ are added. Then the particle size distribution is set to $R=2.5$ and $Q=0.707$, and the concentration is $0.5 \mathrm{~kg} / \mathrm{m}^{3}$. 


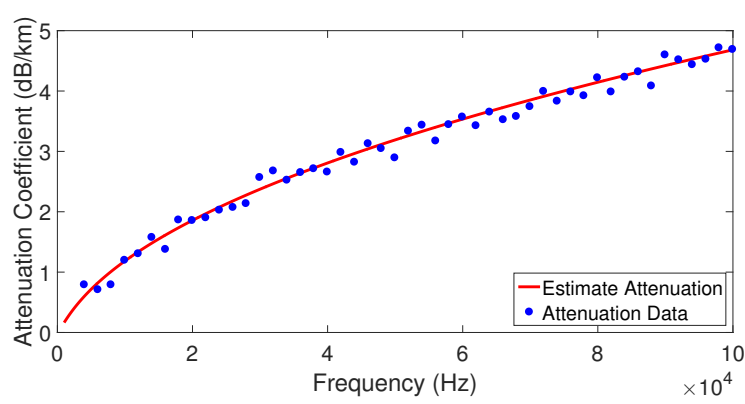

(a) $1 \mathrm{~dB}$ noise

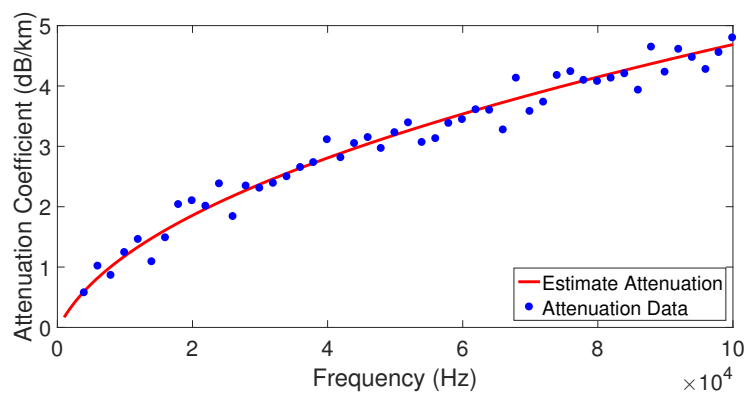

(b) $5 \mathrm{~dB}$ noise

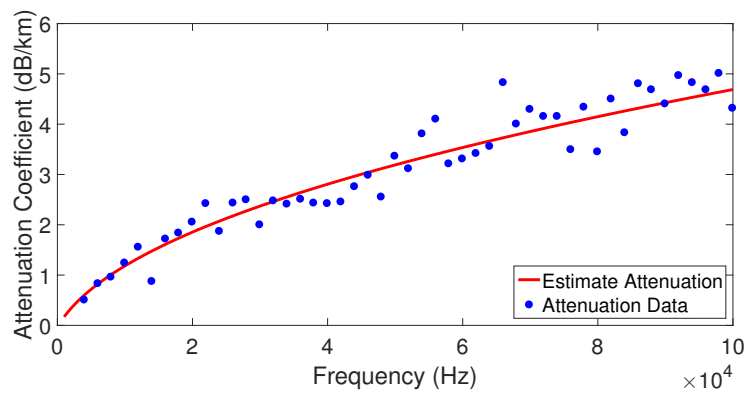

(c)10dB noise

Fig. 7. The estimated attenuation curve calculated by estimated parameters in different noise power.

In Fig 6, the transmitted signal and received signal are shown and an obvious difference is that the amplitude of signals are decreased. In the inversion, a list of results at special steps shows the process that parameters search optimum solution in TABLEI. The values is set as $[C, \bar{\phi}, Q]=[30,-5,2]$ at initial time and approach the real values from fiercely to mildly. Fig 7 shows process that estimation curve is fitting the data from transceiver with different noise power. From TABLEI, the parameters from iteration process when noise power is $1 \mathrm{~dB}$ are closest to the real value. And when noise power is $10 \mathrm{~dB}$, the error can not to be ignored.

\section{CONCLUSION}

In this work, the model of acoustic attenuation in water is considered to measure concentration of particles in suspended water. The simulation contains main factors that cause the decrease of sound power, which are viscous absorption, scattering attenuation, self-absorption of water and geometric attenuation. To achieve accuracy, a Gaussian lognormal distribution of particle size is used to replace the classical average radius. Then, the concentration of particles can be obtained with limited iteration. Further, in the simulation with noise, the error of the parameters obtained by iteration increases with the increase of interference.

\section{REFERENCES}

[1] W.O.C.Jr, B.T.Goodwiller, J.P.Chambers, "Acoustic measurement of suspensions of clay and silt particles using single frequency attenuation and backscatter," Applied Acoustics, 2014, 85:123-129.

[2] A.K.Hipp,S.A.Giuseppe,M.Morbidelli, "Acoustic Characterization of Concentrated Suspensions and Emulsions.1.Model Analysis," Langmuir, 2015, 18(2):391-404.

[3] R.J.Urick, "The Absorption of Sound in Suspensions of Irregular Particles," Journal of the Acoustical Society of America, 1948, 20(3):283289.

[4] J.R.Allegra, S.A.Hawley, "Attenuation of sound in suspensions and emulsions: Theory and experiments: Allegra, J.R.Hawley, S.A. Vol 51 No 5 Part 2 (May 1972) pp 1545C1564," Ultrasonics, 1971, 10(6):287.

[5] S.D.Richards, A.D.Heathershaw, P.D.Thorne, "The effect of suspended particulate matter on sound attenuation in seawater, Journal of the Acoustical Society of America, 1996, 100(3):1447-1450.

[6] P.S.Epstein, R.R.Carhart, "The Absorption of Sound in Suspensions and Emulsions. I. Water Fog in Air," Journal of the Acoustical Society of America, 1948, 20(3):283-289.

[7] J.R.Allegra, S.A.Hawley, G.Holton, "Attenuation of Sound in Suspensions and Emulsions," Journal of the Acoustical Society of America 1972, 48(1A): 1545-1564.

[8] I.N.Mccave, "Vertical flux of particles in the ocean," Deep-Sea Research and Oceanographic Abstracts, 1975, 22(7):491-502.

[9] I.N.Mccave, "Size spectra and aggregation of suspended particles in the deep ocean," Deep Sea Research Part A Oceanographic Research Papers, 1984, 31(4):329-352.

[10] H.Bader, "The hyperbolic distribution of particle sizes," Journal of Geophysical Research, 1970, 75(15):2822-2830.

[11] R.H.Stavn, T.R.Keen, "Suspended minerogenic particle distributions in high-energy coastal environments: Optical implications," Journal of Geophysical Research Oceans, 2004, 109(C5).

[12] M.Schulkin and H.W.Marsh, "Absorption of sound in sea-water," Radio.Electronic Engineer, 1963, 25:493-500.

[13] J.W.S.Rayleigh, R.B.Lindsay, "The theory of Sound," 1945: 15-69.

[14] V.N.Svalnov, T.N.Alekseeva, "Average grain-size parameters of unlithified sediments in the World Ocean," Oceanology, 2006, 46(4):545-556.

[15] S.D.Richards, T.G.Leighton, N.R.Brown, "Sound absorption by suspensions of nonspherical particles: Measurements compared with predictions using various particle sizing techniques," Journal of the Acoustical Society of America, 2003, 114(1):1841-50.

[16] S.A.Moore, C.J.Le, D.Hurther, "Using multi-frequency acoustic attenuation to monitor grain size and concentration of suspended sediment in rivers," Journal of the Acoustical Society of America, 2013, 133(4):1959.

[17] B.D.Moate, P.D.Thorne, "Measurements and inversion of acoustic scattering from suspensions having broad size distributions," Journal of the Acoustical Society of America, 2009, 126(6):2905-17.

[18] M.W.Wedd, "Determination of particle size distributions using laser diffraction," Educational Resources for Particle Technology, 2003, 4(1):14.

[19] J.R.Gray, J.W.Gartner, "Technological advances in suspended-sediment surrogate monitoring," Water Resources Research, 2010, 45(4): 450-455.

[20] J.W.Gartner, "Estimating suspended solids concentrations from backscatter intensity measured by acoustic Doppler current profiler in San Francisco Bay, California," Marine Geology, 2004, 211(3C4): 169-187.

[21] G.Reichel, H.P.Nachtnebel, "Suspended sediment monitoring in a fluvial environment: Advantages and limitations applying an Acoustic Doppler Current Profiler," Water Research, 1994, 28(4):751-761.

[22] I.N.Mccave, "Vertical flux of particles in the ocean," Deep-Sea Research and Oceanographic Abstracts, 1975, 22(7):491-502.

[23] R.H.Stavn, T.R.Keen, " Suspended minerogenic particle distributions in high-energy coastal environments:Optical implications," Journal of Geophysical Research Oceans, 2004, 109(C5). 
[24] Z.Qian, "Concentrated suspension theory of sound attenuation in marine sedimentsłlinear dependence of absorption coefficient on frequency," Journal of Sound, Vibration, 1985, 103(3):427-436. 